\title{
Correlation between Porosity and Electrical-Mechanical Properties of Carbon Nanotube Buckypaper with Various Porosities
}

\author{
Ling Liu, Qiaoxin Yang, and Jingwen Shen \\ School of Aerospace Engineering \& Applied Mechanics, Tongji University, Shanghai 200092, China \\ Correspondence should be addressed to Ling Liu; lingliu@tongji.edu.cn
}

Received 31 August 2015; Revised 6 November 2015; Accepted 19 November 2015

Academic Editor: Albert Nasibulin

Copyright (C) 2015 Ling Liu et al. This is an open access article distributed under the Creative Commons Attribution License, which permits unrestricted use, distribution, and reproduction in any medium, provided the original work is properly cited.

\begin{abstract}
Porous carbon nanotube (CNT) buckypapers (BPs) with various porosities were obtained by using a positive pressure filtration method. The porosity of the BPs fell into a wide range of 11.3-39.3\%. Electrical conductivities and tensile mechanical properties of the prepared BPs were then measured and correlated with the porosity of the CNT BPs. Results demonstrated that the conductivities, tensile strength, and elastic modulus of the BPs could decrease by increasing their porosity. The elongation at break of the BPs on the other hand did increase significantly, suggesting improved toughness of the BPs. The obtained electrical conductivity and tensile strength of the porous BPs can reach nearly $0.6 \mathrm{~S} / \mathrm{m}$ and $26 \mathrm{MPa}$, respectively, which may be potentially useful in composites reinforcement and conductive materials.
\end{abstract}

\section{Introduction}

Buckypaper (BP) is a macroscopic, paperlike nanomaterial composed of continuous entangled carbon nanotubes (CNTs) networks with a highly porous structure, in which the CNTs are freestanding in random direction [1]. The paperlike nanomaterial has several specific features such as lower density, high specific surface area (SSA), high strength to weight ratio, and ability in providing both mechanical and functional properties $[1,2]$. Therefore, CNT BPs can be used for a wide range of applications, such as mechanical reinforcing and functional device fields $[1,2]$.

Typically, CNT BPs are mainly prepared by two kinds of methods, that is, filtration deposition and chemical vaporous deposition (CVD). The filtration deposition method usually involves functionalization of CNTs, dispersing CNTs to obtain stable suspension, filtration under proper pressure, drying, and removal from filtering paper $[1,3]$. The advantages of this method are considered as simple procedures and low equipment cost, but relatively weak mechanical properties of CNT BPs are the main shortcomings $[2,3]$. Relatively high mechanical properties of CNT BPs can be prepared by using one-step CVD method [4], but the weakness of this method is that the size and porosity of CNT BPs are relatively small, needing posttreatment to remove the impurities $[5,6]$. Low porosity of CNT BPs means it is not applicable to the fields of reinforcing materials, especially polymer based composites. Porosity and large size of CNT BPs can be obtained by control of the preparing parameters using filtering method; thus this method is still attracting popular attentions [3].

Up to date, many attempts have been carried out to investigate the electrical and mechanical properties of CNT BPs. The electrical conductivity of CNT BPs published in literatures usually falls into the range of $10^{-3}-10^{7} \mathrm{~S} / \mathrm{m}$ (equivalent value), that is, $186 \Omega / \mathrm{sq}$ [7], $400 \Omega / \mathrm{sq}$ [8], $85 \Omega / \mathrm{sq}$ [9], $3000 \Omega \cdot \mathrm{cm}[10], 1.5 \times 10^{7} \mathrm{~S} / \mathrm{m}[11], 0.005-0.3 \Omega \cdot \mathrm{cm}[12], 10^{-11}$ $10^{-5} \mathrm{~S} \cdot \mathrm{sq}$ [13], $205 \Omega / \mathrm{sq}$ [14], and $1.96 \times 10^{4} \mathrm{~S} / \mathrm{m}$ [15], respectively, reported by Andrade et al. [7], Schindler et al. [8], Yim et al. [9], Wu et al. [10], Lyons et al. [11], Blackburn et al. [12], Simien et al. [13], Ren and Wang [14], and Park et al. [15]. Che et al. [16], Zhang et al. [17], Trakakis et al. [18], Zhang et al. [19], Berhan et al. [20], Steiner et al. [21], Mechrez et al. [22], Sweetman et al. [23], and Špitalský et al. [24] reported that the tensile strength and modulus of BPs are measured among 2$35 \mathrm{MPa}$ and $0.2-5 \mathrm{GPa}$, respectively, whereas the elongation at break commonly varies between $0.5 \%$ and $4 \%$ [3]. 
Although remarkable physical properties have been achieved by use of this method, bulk mechanical properties of CNT BPs remain relatively low. The reported values fall into a wide range; this is because the conductivities and mechanical properties of BPs depend on not only the intrinsic electrical and mechanical properties of CNTs, but also the geometries and chemical treatment of the used CNTs, especially the microstructure of the prepared BPs. CNTs commonly random align in the $\mathrm{BP}$ and freely stack one over another by Van der Waals force; this may be the most important reason that affects the physical and mechanical properties. Some literatures have discussed the effect of alignment and density of CNTs in BPs on the properties of BPs $[11,16,19,20]$, but detailed microstructure, such as porosity and pores size, has seldom been addressed. More efforts, especially microstructure studies of BPs and corresponding mechanism, are needed to explore the influencing factors so as to fulfill this relatively young research field.

To obtain the relationship between properties and microstructure of CNT BPs, this paper aimed to prepare various porous BPs by using a simple and convenient positive filtering method. SEM, FTIR, Raman spectrum, and TEM analyses were used to evaluate the morphology and microstructure of the obtained BPs. Pores size and distribution, porosity, and SSA were evaluated by using surface area and porosity analyzer method. Based on the microstructure analysis, electrical conductivity and mechanical properties of the prepared BPs with various porosities were examined by resistivity measurement and tensile testing methods, respectively. By varying the porosity of CNT BPs, the conductivity and mechanical properties of the CNT BPs were correspondingly changed as expected. The emphasis of this study is correlation of the properties and the microstructure of the BPs, instead of emphasizing the absolute value of the conductivity and mechanical properties of the BPs.

\section{Experimental}

\subsection{Preparation}

2.1.1. Materials. Multiwalled CNTs $(8 \sim 15 \mathrm{~nm}$ in diameter and $5 \sim 15 \mu \mathrm{m}$ in length) used in this study were purchased from Chengdu Organic Chemicals Co. The CNTs were obtained as suspension with a concentration of acid oxidation treated CNTs 2 wt.\% in dimethylformamide (DMF) solution. The ratio of the functionalized CNTs was nearly $1.85 \mathrm{wt} . \%$. The conductivity of the original CNTs (before dispersion) was nearly $10^{5} \mathrm{~S} / \mathrm{m}$ (supplied by the manufacturer). Other reagents include concentrated nitric acid and deionized water.

2.1.2. Preparation of CNT BPs. The CNTs suspension was filtrated through a polytetrafluoroethylene (PTFE) membrane with a pore size of $0.22 \sim 1.2 \mu \mathrm{m}$ under an aid of positive pressure $(0.05 \sim 0.2 \mathrm{MPa})$. The preparing parameters, such as concentration of CNTs suspension, the applied pressure, pores diameter of PTFE, and postprocessing time by using nitric acid to remove surfactant, will have somewhat effect on the porosity of the BPs according to our previous study. Thus,
TABLE 1: The prepared BPs with various preparation parameters.

\begin{tabular}{lccc}
\hline BPs & $\begin{array}{c}\text { Pore diameter in PTFE } \\
\mu \mathrm{m}\end{array}$ & $\begin{array}{c}\text { Filtration pressure } \\
\mathrm{MPa}\end{array}$ & $\begin{array}{c}\text { Thickness } \\
\mathrm{mm}\end{array}$ \\
\hline $1^{\#}$ & 0.22 & 0.05 & 0.038 \\
$2^{\#}$ & 0.8 & 0.05 & 0.034 \\
$3^{\#}$ & 1.2 & 0.05 & 0.036 \\
\hline
\end{tabular}

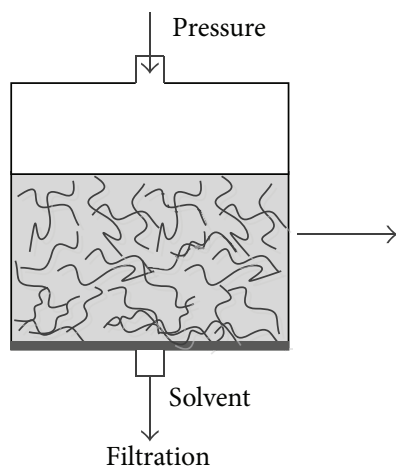

(a)

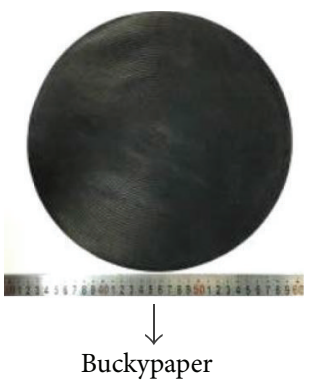

(b)
FIGURE 1: Schematic of positive pressure preparation of CNT BP (a) and the final obtained BP (b).

to obtain various porosities of the BPs, the concentration of CNTs in suspensions and the applied pressure were kept the same and we mainly changed the diameters of the filter membrane to avoid too many variables (shown in Table 1). Three kinds of porosities of the BPs, named $1^{\#}, 2^{\#}$, and $3^{\#}$, were prepared by changing the diameters of the PTFE as $0.22,0.8$, and $1.2 \mu \mathrm{m}$, respectively. The CNT suspensions were firstly filled into the filtering tank (sketch map showed in Figure 1(a)), and then we applied positive pressure by an air compressor. The solvent in the suspensions was squeezed out and we left the CNTs on the PTFE membrane. The obtained CNT BPs were then immersed into nitric acid for $5 \mathrm{~min}$ to remove surfactant. Then, the paper films were washed with distilled water to remove impurities and acid and were directly dried in a bake oven. Finally, the CNT films were peeled from the PTFE membrane and the BP products were showed in Figure 1(b). The BPs were circular with a thickness about $0.03-0.04 \mathrm{~mm}$ (measured by a micrometer) and a diameter of $280 \mathrm{~mm}$ (indicated in Table 1).

\subsection{Characterization}

2.2.1. Morphology and Microstructure. The final morphologies of the BPs were observed with a SEM machine (Philips XL 30 ESEM instrument). The samples with various porosities were observed for a comparison purpose. Fourier transform infrared (FTIR) for the prepared BP was obtained (Nicolet 6700 FTIR spectrometer using attenuated total transmission mode) to investigate the functional groups on the walls of CNTs. To evaluate the structure integrity, Raman spectrum of the BP (rectangular beam with dimensions of $5 \times$ $5 \times 0.035 \mathrm{~mm}^{3}$ ) was obtained using a XploRA Laser Raman 


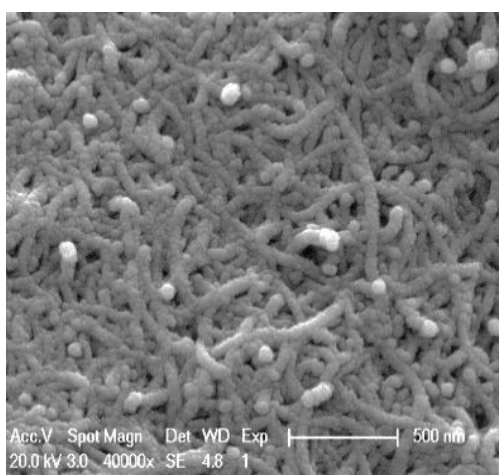

(a)

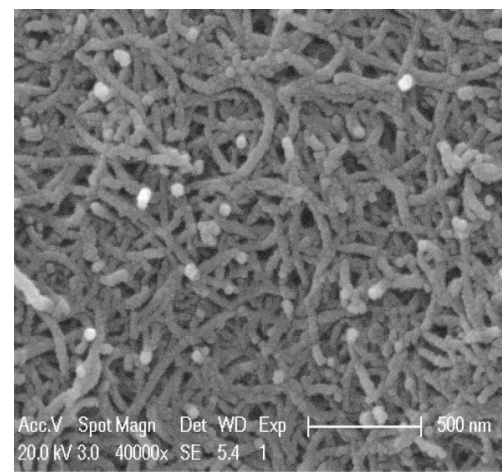

(b)

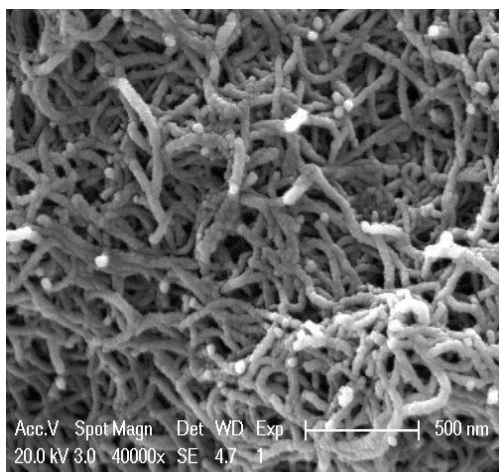

(c)

FIGURE 2: SEM images of the BPs with various porosities, (a), (b), and (c) denoting $1^{\#}, 2^{\#}$, and $3^{\#}$ BPs, respectively.

Spectrometer (HORIBA Jobin Yvon, France) with $638 \mathrm{~nm}$ red line of a He-Ne laser and a charged coupled device (CCD) as a detector. A 50x objective lens was used to focus the laser beam on sample surface (laser spot: $1.56 \mu \mathrm{m}$ ). TEM (JEM 2011, Jeol, Japan) was used to analyze the crystallinity and microstructure. Nitrogen adsorption-desorption isotherms were measured at $77 \mathrm{~K}$ on a QuadraSorb SI surface area and porosity analyzer (Quantachrome, USA). The SSA was calculated by using Brunauer-Emmett-Teller (BET) method from the adsorption branch of the $\mathrm{N}_{2}$ adsorption isotherm, while the pore size and pore size distribution were evaluated using Barrett-Joyner-Halenda (BJH) analysis from the $\mathrm{N}_{2}$ desorption isotherm [25].

2.2.2. Electrical Conductivity. Electrical conductivity of all final BPs was measured using an Agilent 4294A impedance analyzer in the frequency range of $40 \mathrm{~Hz}$ to $100 \mathrm{MHz}$ with an $\mathrm{AC}$ voltage of $500 \mathrm{mV}$. Complex impedance modulus, $Z$, of all samples at different frequency can be obtained from the analyzer. Therefore, electrical conductivity, $\sigma$, can be calculated from the formula

$$
\sigma=\left(\frac{1}{Z}\right) *\left(\frac{t}{A}\right)
$$

where $t$ is the thickness of the BPs and $A$ is the area of the electrode (whose diameter is $38 \mathrm{~mm}$ ). The two end surfaces of all samples were coated with conductive adhesive for ohmic contact with the electrode. For evaluating the repeatability, three to five specimens were tested.

2.2.3. Mechanical Properties. To characterize the tensile performance of the BPs, a micro tensile testing machine was used (BT1-FPLV.00, Zwick/Roell, Germany), equipped with a $500 \mathrm{~N}$ load cell. The test specimens were cut into dumbbell shape with a length of $38 \mathrm{~mm}$, and the gauge length and width were $15 \mathrm{~mm}$ and $5 \mathrm{~mm}$, respectively, according to ASTM D882-10 standard. The tensile strain was measured using a noncontact method, a laser speckle measuring apparatus, with a $4 \mathrm{~mm}$ gauge length. During the testing, the applied crosshead speed was set as $1 \mathrm{~mm} / \mathrm{min}$. Again, three to five specimens were measured to evaluate the consistency of the results.

\section{Results and Discussion}

3.1. Morphology and Microstructure. SEM analysis of the BPs with various porosities was provided in Figure 2, where (a), (b), and (c) indicate the $1^{\#}, 2^{\#}$, and $3^{\#}$ BPs, respectively. It was clearly shown from Figure 2(a) that the $1^{\#}$ BP was relatively dense and smooth with small pores that could be seen. The $2^{\#} \mathrm{BP}$ showed larger pores compared with that of the $1^{\#} \mathrm{BP}$ (Figure 2(b)), and the surface also looked smooth. But the $3^{\#}$ BP looked loose and uneven, indicating larger and more pores in the views (Figure 2(c)). Therefore, the SEM observations preliminarily proved various porosities of BPs were obtained by varying the diameters of the filter membranes. Moreover, these three BPs were composed of randomly distributed CNTs from the viewpoint of microphotograph.

Figure 3(a) presented the FTIR spectrum of the CNT papers. The presence of characteristic absorption peak for $\mathrm{O}-\mathrm{H}$ group (nearly at $3410 \mathrm{~cm}^{-1}$ ) and $\mathrm{C}=\mathrm{O}$ (in $-\mathrm{COOH}$ group, nearly at $1615 \mathrm{~cm}^{-1}$ ) clearly stated the existing of carboxyl group and hydroxyl group after functionalization treating. The Raman spectrum of the obtained BP was given in Figure 3(b). For CNT, its intensity ratio of defect mode (D) to tangential shear mode $(\mathrm{G}), I_{\mathrm{D}} / I_{\mathrm{G}}$, can be utilized to qualitatively characterize its defect density [26]. It was found from Figure 3(b) that the $I_{\mathrm{D}} / I_{\mathrm{G}}$ value of CNTs in the BP was nearly equal to 1.50 . This result confirms the fact that the integrity of CNTs was not destroyed after oxidation treatment and thus their mechanical and physical properties would be maintained to some degree [27]. The TEM micrograph (Figure 3(c)) suggested that some CNTs kept relatively good structural integrity after chemical functionalization. But the crystallinity of some CNTs was still degraded due to the oxidation treatment, proving by the unsmooth surface or defects of CNTs [28].

The measured pore diameters and distributions of the BPs by using BJH method were given in Figure 4. It showed the diameters of pores are mainly distributed between 10 and $30 \mathrm{~nm}, 10$ and $50 \mathrm{~nm}$, and 20 and $60 \mathrm{~nm}$ for the $1^{\#}, 2^{\#}$, and $3^{\#}$ BPs, suggesting average diameters of $19 \mathrm{~nm}, 31 \mathrm{~nm}$, and $40 \mathrm{~nm}$ from the fitting curves, respectively. The average diameters of the pores, open porosity, and SSA (by BET method) for these three BPs were also reported in Table 2. 
TABLE 2: Microstructures and pores size of the obtained BPs.

\begin{tabular}{lccccc}
\hline \multirow{2}{*}{ BPs } & \multicolumn{2}{c}{ Diameter of pores $(\mathrm{nm})$} & Porosity $(\%)$ & SSA $\left(\mathrm{m}^{2} / \mathrm{g}\right)$ & Density $\left(\mathrm{g} / \mathrm{cm}^{3}\right)$ \\
\hline $1^{\#}$ & Average & SD & 11.3 & 156 & 0.44 \\
$2^{\#}$ & 19 & 11 & 21.1 & 189 & 0.38 \\
$3^{\#}$ & 31 & 19 & 39.3 & 197 & 0.29 \\
\hline
\end{tabular}

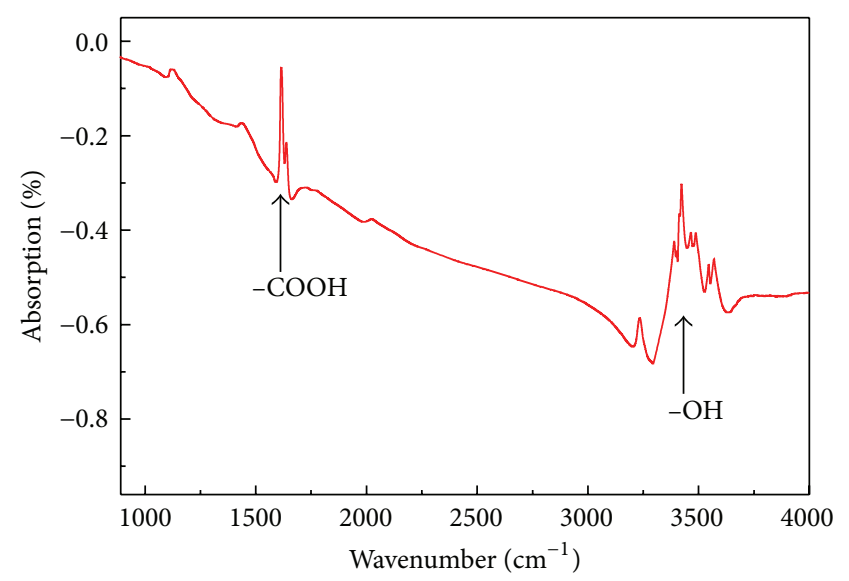

(a)

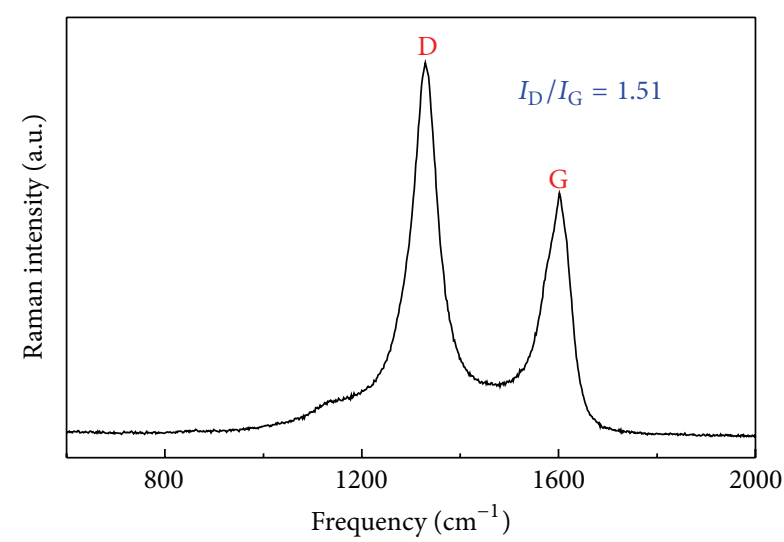

(b)

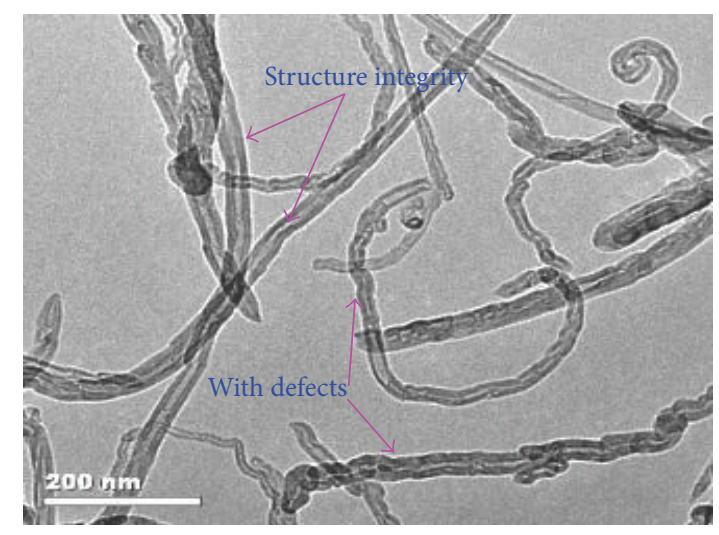

(c)

FIgURE 3: Microstructural information of the used CNTs, (a) FTIR spectrum, (b) Raman spectrum, and (c) TEM micrograph.

The porosities of the $1^{\#}, 2^{\#}$, and $3^{\#}$ BPs were measured as 11.3 , 21.1, and $39.3 \%$, respectively. Also, the SSA for these three BPs were correspondingly calculated as 156,189 , and $197 \mathrm{~m}^{2} / \mathrm{g}$. Improvements up to $87 \%$ and $248 \%$ for the porosity and $20 \%$ and $26 \%$ for the SSA were, respectively, obtained for the $2^{\#}$ and $3^{\#}$ BPs compared with those of the $1^{\#}$ BPs. Moreover, the densities of the BPs were decreased with the increasing of the porosities of the BPs (Table 2). It can be concluded from Figure 4 and Table 2 that the porosities of the BPs were increased with the increasing of the pores size, suggesting the preparation parameters have significant effect on the pores size and porosity of the BPs by using filtration method.

3.2. Conductive Properties. Figure 5(a) showed the frequency dependence of the electrical conductivities of the BPs with various porosities. The testing specimens exhibited a directcurrent conductivity plateau in the whole frequency range, where the conductivity is independent of frequency. According to (1), the conductivity $(\sigma)$ was correlated with the complex impedance modulus $(Z)$ for the same specimen $(t / A$ is constant), while $Z$ is calculated from

$$
Z=R+j X
$$

where the real part $R$ is the resistance, which is independent of frequency. The imaginary part $X$ is the sum of capacitance and inductive reactance, which is dependent on the frequency. The imaginary part $X$ was always tested as zero in our testing. Therefore, the BPs exhibited feature of direct-current conductive materials and resulted in a plateau behavior in the whole frequency range showed in Figure 5(a). The correlation 


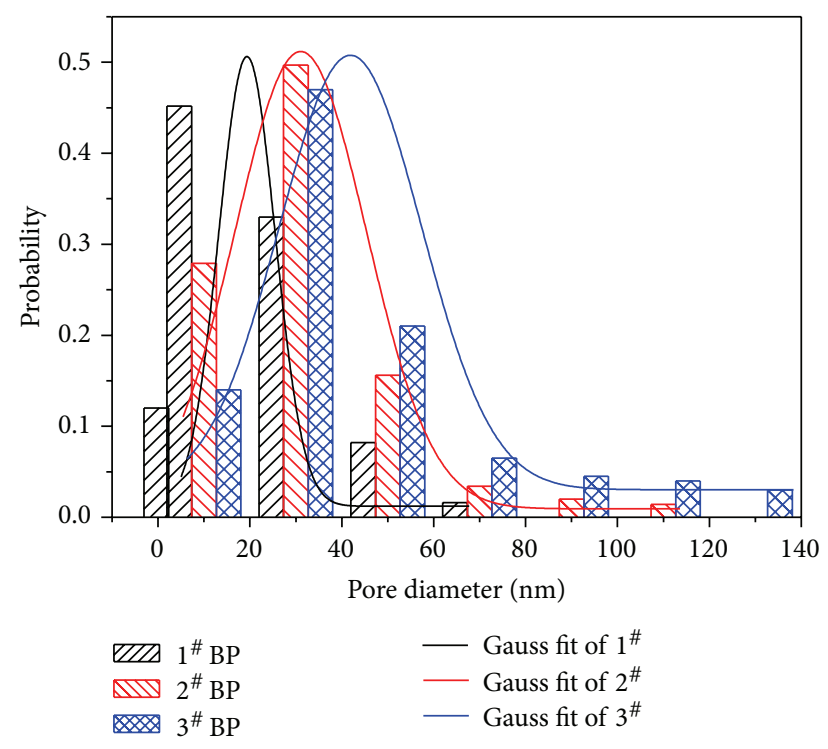

FIGURE 4: Measured distribution of pores diameter of the $1^{\#}, 2^{\#}$, and $3^{\#}$ BPs.

between conductivity and porosity of the BPs was illustrated in Figure 5(b). As was expected, the conductivities were decreased with the increasing of the porosity of the BPs (Figure 5(b)). The $1^{\#}$ BP showed higher conductivities of $0.631 \mathrm{~S} / \mathrm{m}$ due to its small porosity of $11.3 \%$. The conductivity of the $2^{\#}$ BP was reduced to $0.086 \mathrm{~S} / \mathrm{m}$ (Figure 5(b)), with a decrease of nearly ten times compared with that of the $1^{\#}$ $\mathrm{BP}$. The same trend was also obtained for the $3^{\#} \mathrm{BP}$, with an average conductivity of $0.011 \mathrm{~S} / \mathrm{m}$. It meant that the porosity had significant effect on the conductivity of the porous BPs. It can be seen from Figure 5 that the obtained conductivities of the BPs belong to the range of semiconductive materials; therefore, the BPs prepared by filtering method have potential applications such as electromagnetic shielding, electric battery, and sensor.

Here a short discussion on conductive mechanism for the BPs was given and showed in Figure 6. For direct-current based conductive materials like the CNT BPs, the conductivity of the BPs mainly originates from ohmic conduction mechanism, through direct contact of conductive CNTs. As can be seen from Figure 2, the individual CNTs were randomly stacked in BPs by Van der Waals force between adjacent CNTs. The "contacting" of CNTs in BPs can be mainly divided into three patterns, that is, body-to-body (Figure 6(a) (1)), body-to-end (Figure 6(a) (2)), and endto-end (Figure 6(a) (3)), as shown in Figure 6(a) by TEM observation. Suppose each CNT acted as a conductor and the current mainly flowed along the axis of CNTs (indicated in Figure 6(b)). The current will be branched at the contact point as well as be weakened by the contact resistance (Figure 6(b)). If these three contact resistances were supposed to be equivalent resistances shown as in Figure 6(b), thus mixed conduction paths, including parallel connection and series connection, were formed (partly supposed as in Figure 6(c)). When the porosity of the BPs is small, the number of

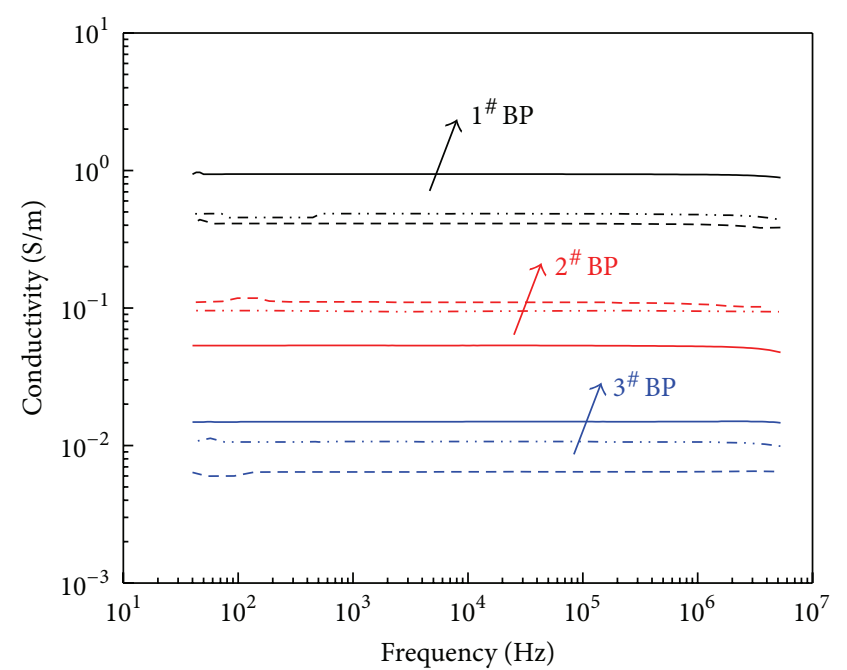

(a)

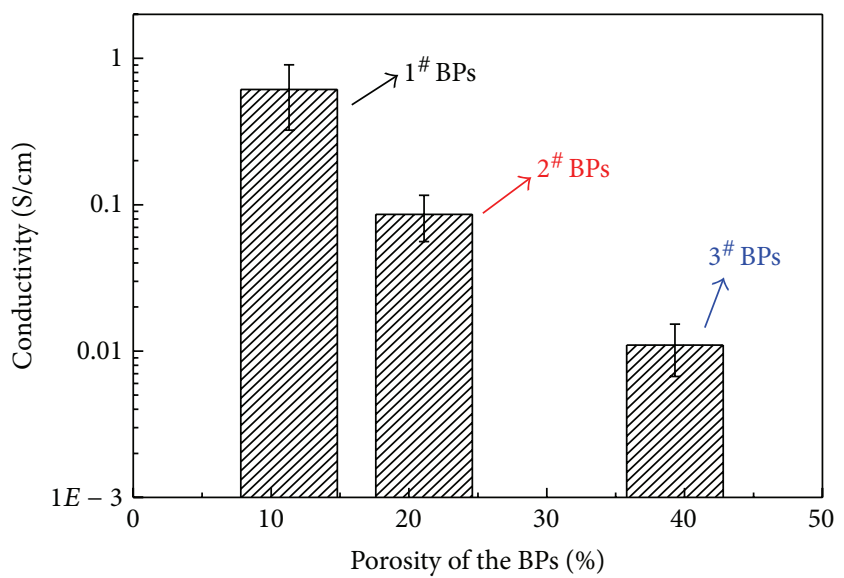

(b)

FIgURE 5: The conductivities of the tested BPs versus the frequency (a) and the correlation between the porosity and conductivity of the BPs (b).

the CNTs in the BPs will be larger, including more short CNTs. More CNTs will contact each other and thus more conductive paths and larger effective conductive area were formed through the thickness of the BPs. It can be imagined that more free electrons in the inner of the CNTs tubes will form directional movement when applying constant outer AC voltage. Therefore, the resistance will decrease according to Ohm's law, and the conductivity will increase accordingly.

It is worth noting that the obtained conductivity of the CNT BPs was smaller than that of several remarkable reported results recorded as $10^{4} \mathrm{~S} / \mathrm{m}$ [11] and $10^{7} \mathrm{~S} / \mathrm{m}$ [15]. On one hand, high conductivity of BPs results from high alignment and high density (such as $1.39 \mathrm{~g} / \mathrm{cm}^{3}$ ) of CNTs [16], while the BPs in our study are porous and the CNTs are randomly stacked in the BPs with low density (0.3$0.4 \mathrm{~g} / \mathrm{cm}^{3}$ ). On the other hand, the chemical functionalization significantly has effect on the conductivity of the CNTs, which further affects the conductivity of the BPs. The CNTs in our study are functionalized by carboxyl and 


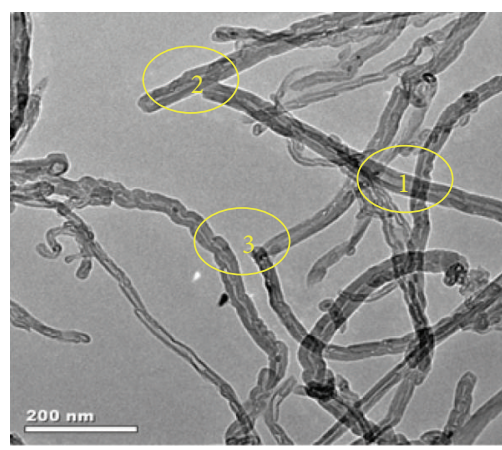

(a)

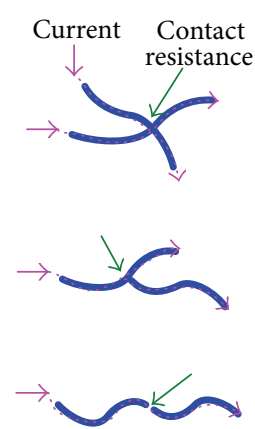

Equivalent resistance
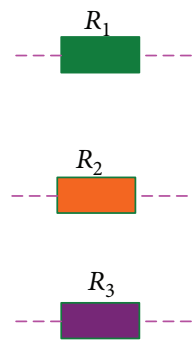

(b)

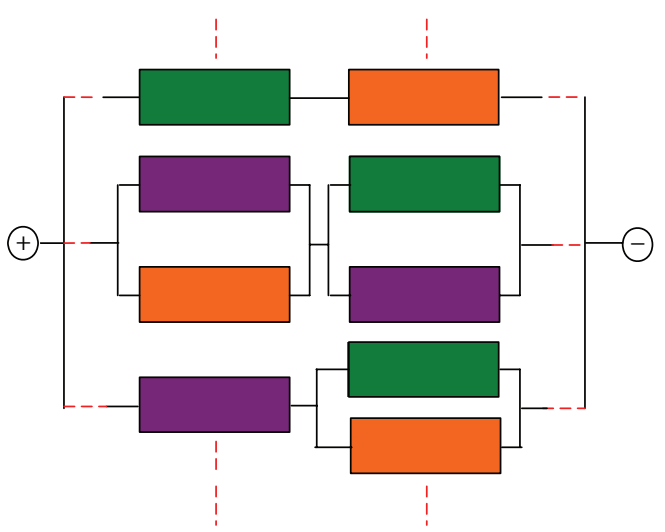

(c)

FIGURE 6: The contact patterns of CNTs in the BPs indicated by TEM observation (a), where (1), (2), and (3) denote body-to-body, body-to-end, and end-to-end patterns, respectively, contact patterns and corresponding equivalent resistance (b), and the partial sketch map of the possible existing of mix conduction paths in the BPs (c).

hydroxyl groups, which decreased the bulk conductivity of the CNTs. Furthermore, in calculated formula (1), $A$ is the area of the measured electrode (also used as the tested area of the specimen), but the effective area of the BPs is smaller than the measured area due to the pores and porosity of the BPs. Therefore, these factors lead to a relatively smaller conductivity of the prepared BPs in this research.

3.3. Mechanical Properties. Typical tensile stress-strain curves for $1^{\#}, 2^{\#}$, and $3^{\#}$ BPs were given in Figure 7(a). All the samples showed nonlinear and nearly bilinear behavior, especially the $2^{\#}$ and $3^{\#}$ BPs, with significant inflection point in the curves (Figure $7(\mathrm{a})$ ). The $1^{\#}$ BPs showed a shorter elongation at break about $2.2 \%$, which was smaller than that of the $2^{\#}$ and $3^{\#}$ BPs, calculated as $3.5 \%$ and $5.2 \%$, respectively. With the increasing of the porosity of the BPs, the nonlinear behavior and elongation were both increased significantly, suggesting the flexibility or ductility became better. The tensile strength of $1^{\#}, 2^{\#}$, and $3^{\#}$ was measured as 23.8, 25.5, and 21.8 MPa, respectively (shown in Figure 7(b)). Compared to the $1^{\#}$ BPs, the tensile strength of the $2^{\#}$ BPs increased by $7 \%$ and decreased by $8 \%$ for the $3^{\#}$ BPs. The obtained relatively good tensile strength proved that efficient load transfer in the BPs was formed. The tensile elastic modulus was decreased with the porosity increasing of the BPs. As was shown in Figure 7(b), the modulus of the $2^{\#}$ BPs reduced to $1.64 \mathrm{GPa}$ with respect to $\mathrm{l}^{\#} \mathrm{BPs}(2.11 \mathrm{GPa})$, with a decrease of $22 \%$. A similar trend was found for the $3^{\#}$ BPs, with a decrease of modulus $43 \%$ when the porosity of the BPs increased from $11.3 \%$ to $39.3 \%$. The tensile properties were coherent with the reported range of literatures [19-21], especially the tensile strength which was close to the reported maximum value. The obtained good mechanical properties of the BPs with functional groups mean that it can be used for reinforced materials.

It can be deduced that CNTs in the BPs are randomly oriented; as mentioned before, the individual CNTs were stacked one over another just by "contacting" pattern and Van der Waals force between adjacent CNTs. However, the CNTs themselves and the Van der Waals force between CNTs both contributed to the load bearing. Stretch of CNTs and slippage between CNTs mainly contributed to the deformation or strain. The CNT BPs may undergo three main behaviors under outer tensile stress. Firstly, in the initial stage of applied load, the applied load was quickly transferred to adjacent CNTs; the CNTs bore the load and extended (see in Figure 8(a)), so the stress was linearly increased with the strain (showed in Figure 7(a)). Due to the small porosity of the $1^{\#}$ BPs, maybe more CNTs could contact each other and lie in the BPs; thus more load can be born, resulting in quicker increasing of stress and larger elastic modulus for the $1^{\#}$ BPs (due to the same strain rate for all specimens). 


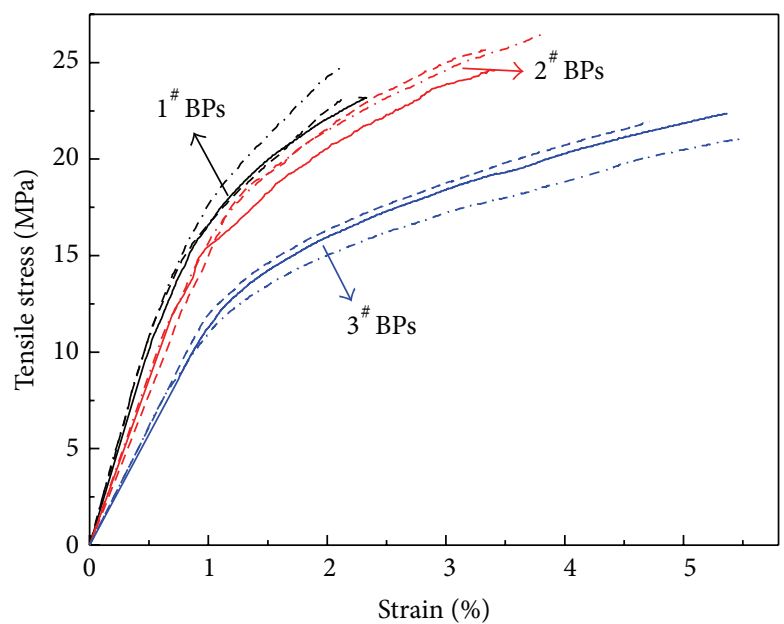

(a)

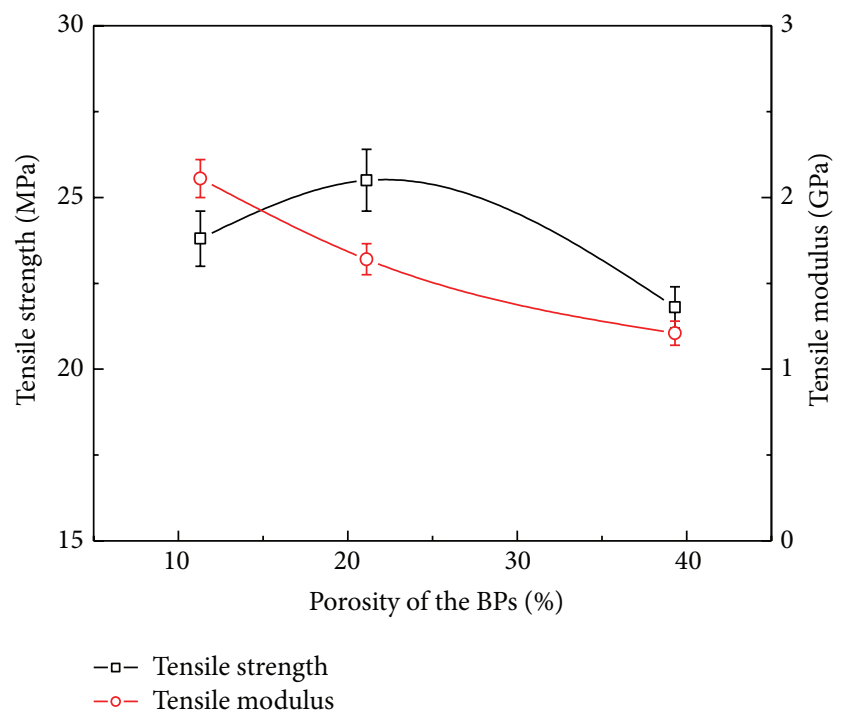

(b)

FIgURE 7: Tensile stress-strain curves of the prepared BPs (a) and correlation between porosity and tensile properties (b).

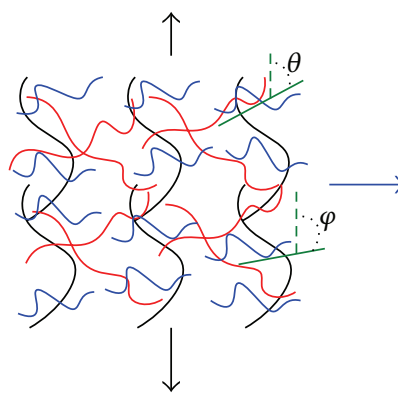

(a)

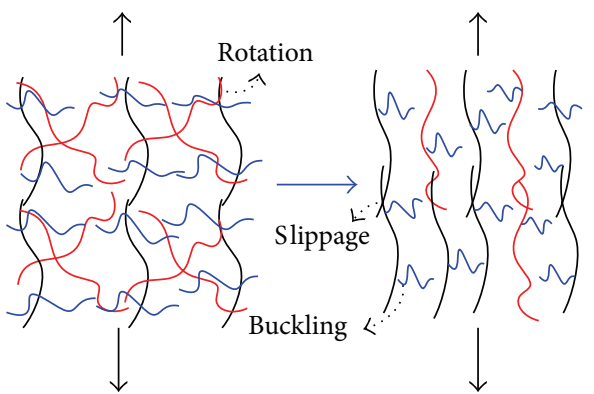

(b)

(c)

FIgURE 8: Tensile behaviors of the prepared BPs, (a) the initial linear stage, (b) inflection region, and (c) final stage of the tension.

With the increasing of porosity and decreasing number of effective bearing CNTs, the modulus was decreased in the initial stage of stress-strain behavior for the $2^{\#}$ and $3^{\#}$ BPs because the applied strain rate was the same as that of the $1^{\#}$ BPs. In the second stage of inflection point, some complicated processes may occur. CNTs oriented parallel to the direction of applied strain will continually be stretched and bear the load. CNTs oriented at smaller angle (such as $\theta$ in Figure 8(a)) with respect to the direction of applied strain will be rotated and then be stretched to carry some loads. CNTs oriented at larger angle (such as $\varphi$ in Figure 8(a)) with the direction of the applied strain, as well as approximately perpendicular to the direction of applied strain, will buckle and contribute zero to the total stress in the BPs. Therefore, the increasing rate of stress began to reduce due to the decreasing amount of effective loading CNTs (indicated in Figure 8(b)). At the third linear stage of the stress-strain curves shown as in Figure 7(a), mainly the remainder CNTs parallel to the direction of applied strain would bear the load, and the continual stretch of CNTs and slippage between CNTs would contribute to the deformation, as shown in Figure 8(c). The slippage between CNTs will last until the breakage of the BPs.
There are many shorter CNTs in the $1^{\#}$ BPs with smaller porosity; the stretch and slippages between adjacent CNTs are limited. Thus, fracture occurs quickly and the strength is relatively weak. With similar thickness and larger porosity, the $2^{\#}$ BP possesses more longer CNTs, the stretch and slippages between longer CNTs would last a longer time, and the BP can bear the increasing load at the same time. Therefore, the strength is relatively larger than that of the $1^{\#}$ BP (showed in Figure 7(b)). With increasing of porosity, the number of CNTs in the BP decreased significantly, which led to decrease of strength.

\section{Conclusions}

In summary, CNT BPs with various porosities were successfully prepared by using positive pressure filtration method. Electrical and mechanical tests on CNT BPs revealed that the properties of the CNTs were highly dependent on the pores and porosities of the BPs. The electrical conductivity of the BPs showed behaviors of conductive materials and decreased with the increasing of the porosity. Tensile strength and modulus were basically reduced with the increasing of porosity. 
The elongation at break on the other hand did increase significantly. The highest tensile strength and electrical conductivity were measured as $26 \mathrm{MPa}$ and $0.6 \mathrm{~S} / \mathrm{m}$, respectively. Some relative mechanics correlating the properties and microstructure were somewhat discussed and revealed. These results provide a solid foundation for further investigating CNT BPs for reinforcing and conductive materials applications.

\section{Conflict of Interests}

The authors declare that there is no conflict of interests regarding the publication of this paper.

\section{Acknowledgments}

The project was supported by the Natural Science Foundation of China (Grant no. 11172211). Support from mechanical testing and materials characterization is appreciated. The authors would like to acknowledge Master Degree Candidate Lulu Shen for positive contributions, and the authors also acknowledge Yiwen Zhou for useful advice and help.

\section{References}

[1] L. Hu, D. S. Hecht, and G. Grüner, "Carbon nanotube thin films: fabrication, properties, and applications," Chemical Reviews, vol. 110, no. 10, pp. 5790-5844, 2010.

[2] Z. Wang, Z. Y. Liang, B. Wang, C. Zhang, and L. Kramer, "Processing and property investigation of single-walled carbon nanotube (SWNT) buckypaper/epoxy resin matrix nanocomposites," Composites Part A: Applied Science and Manufacturing, vol. 35 , no. 10, pp. 1225-1232, 2004.

[3] J.-H. Han, H. Zhang, M.-J. Chen, G.-R. Wang, and Z. Zhang, "CNT buckypaper/thermoplastic polyurethane composites with enhanced stiffness, strength and toughness," Composites Science and Technology, vol. 103, pp. 63-71, 2014.

[4] Q. F. Cheng, J. P. Wang, K. L. Jiang, Q. Q. Li, and S. S. Fan, "Fabrication and properties of aligned multiwalled carbon nanotube-reinforced epoxy composites," Journal of Materials Research, vol. 23, no. 11, pp. 2975-2983, 2008.

[5] Q. F. Cheng, J. W. Bao, J. G. Park, Z. Y. Liang, C. Zhang, and B. Wang, "High mechanical performance composite conductor: multi-walled carbon nanotube sheet/bismaleimide nanocomposites," Advanced Functional Materials, vol. 19, no. 20, pp. 32193225, 2009.

[6] Q. F. Cheng, B. Wang, C. Zhang, and Z. Y. Liang, "Functionalized carbon-nanotube sheet/bismaleimide nanocomposites: mechanical and electrical performance beyond carbon-fiber composites," Small, vol. 6, no. 6, pp. 763-767, 2010.

[7] M. J. Andrade, M. D. Lima, V. Skákalová, C. P. Bergmann, and S. Roth, "Electrical properties of transparent carbon nanotube networks prepared through different techniques," Physica Status Solidi, vol. 1, no. 5, pp. 178-180, 2007.

[8] A. Schindler, J. Brill, N. Fruehauf, J. P. Novak, and Z. Yaniv, "Solution-deposited carbon nanotube layers for flexible display applications," Physica E: Low-Dimensional Systems \& Nanostructures, vol. 37, no. 1-2, pp. 119-123, 2007.

[9] J. H. Yim, Y. S. Kim, K. H. Koh, and S. Lee, "Fabrication of transparent single wall carbon nanotube films with low sheet resistance," Journal of Vacuum Science and Technology B:
Microelectronics \& Nanometer Structures, vol. 26, no. 2, pp. 851855, 2008.

[10] Z. Wu, Z. Chen, X. Du et al., "Transparent, conductive carbon nanotube films," Science, vol. 305, no. 5688, pp. 1273-1276, 2004.

[11] P. E. Lyons, S. De, F. Blighe et al., "The relationship between network morphology and conductivity in nanotube films," Journal of Applied Physics, vol. 104, no. 4, Article ID 044302, 8 pages, 2008.

[12] J. L. Blackburn, T. M. Barnes, M. C. Beard et al., "Transparent conductive single-walled carbon nanotube networks with precisely tunable ratios of semiconducting and metallic nanotubes," ACS Nano, vol. 2, no. 6, pp. 1266-1274, 2008.

[13] D. Simien, J. A. Fagan, W. Luo, J. F. Douglas, K. Migler, and J. Obrzut, "Influence of nanotube length on the optical and conductivity properties of thin single-wall carbon nanotube networks," ACS Nano, vol. 2, no. 9, pp. 1879-1884, 2008.

[14] L. Q. Ren and S. R. Wang, "Tailoring optical and electrical properties of carbon nanotube networks for photovoltaic applications," Carbon, vol. 48, no. 15, pp. 4397-4402, 2010.

[15] E. J. Park, K.-D. Kim, H. S. Yoon et al., "Fabrication of conductive, transparent and superhydrophobic thin films consisting of multi-walled carbon nanotubes," RSC Advances, vol. 4, no. 57, pp. 30368-30374, 2014.

[16] J. Che, P. Chen, and M. B. Chan-Park, "High-strength carbon nanotube buckypaper composites as applied to free-standing electrodes for supercapacitors," Journal of Materials Chemistry A, vol. 1, no. 12, pp. 4057-4066, 2013.

[17] L. Zhang, G. Zhang, C. Liu, and S. Fan, "High-density carbon nanotube buckypapers with superior transport and mechanical properties," Nano Letters, vol. 12, no. 9, pp. 4848-4852, 2012.

[18] G. Trakakis, D. Tasis, C. Aggelopoulos, J. Parthenios, C. Galiotis, and K. Papagelis, "Open structured in comparison with dense multi-walled carbon nanotube buckypapers and their composites," Composites Science and Technology, vol. 77, pp. 52-59, 2013.

[19] X. Zhang, T. V. Sreekumar, T. Liu, and S. Kumar, "Properties and structure of nitric acid oxidized single wall carbon nanotube films," Journal of Physical Chemistry B, vol. 108, no. 42, pp. 16435-16440, 2004.

[20] L. Berhan, Y. B. Yi, A. M. Sastry, E. Munoz, M. Selvidge, and R. Baughman, "Mechanical properties of nanotube sheets: alterations in joint morphology and achievable moduli in manufacturable materials," Journal of Applied Physics, vol. 95, no. 8, pp. 4335-4345, 2004.

[21] S. Steiner, S. Busato, and P. Ermanni, "Mechanical properties and morphology of papers prepared from single-walled carbon nanotubes functionalized with aromatic amides," Carbon, vol. 50, no. 5, pp. 1713-1719, 2012.

[22] G. Mechrez, R. Y. Suckeveriene, R. Tchoudakov, A. Kigly, E. Segal, and M. Narkis, "Structure and properties of multi-walled carbon nanotube porous sheets with enhanced elongation," Journal of Materials Science, vol. 47, no. 16, pp. 6131-6140, 2012.

[23] L. J. Sweetman, L. Nghiem, I. Chironi, G. Triani, M. In Het Panhuis, and S. F. Ralph, "Synthesis, properties and water permeability of SWNT buckypapers," Journal of Materials Chemistry, vol. 22, no. 27, pp. 13800-13810, 2012.

[24] Z. Špitalský, C. Aggelopoulos, G. Tsoukleri et al., "The effect of oxidation treatment on the properties of multi-walled carbon nanotube thin films," Materials Science and Engineering B, vol. 165 , no. 3, pp. 135-138, 2009.

[25] E. P. Barrett, L. G. Joyner, and P. P. Halenda, "The determination of pore volume and area distributions in porous substances. 
I. Computations from nitrogen isotherms," Journal of the American Chemical Society, vol. 73, no. 1, pp. 373-380, 1951.

[26] M. E. Itkis, D. E. Perea, R. Jung, S. Niyogi, and R. C. Haddon, "Comparison of analytical techniques for purity evaluation of single-walled carbon nanotubes," Journal of the American Chemical Society, vol. 127, no. 10, pp. 3439-3448, 2005.

[27] Z. Q. Zhang, B. Liu, Y. L. Chen, H. Jiang, K. C. Hwang, and Y. Huang, "Mechanical properties of functionalized carbon nanotubes," Nanotechnology, vol. 19, no. 39, Article ID 395702, 2008.

[28] A. C. Dillon, T. Gennett, K. M. Jones, J. L. Alleman, P. A. Parilla, and M. J. Heben, "A simple and complete purification of singlewalled carbon nanotube materials," Advanced Materials, vol. 11, no. 16, pp. 1354-1358, 1999. 

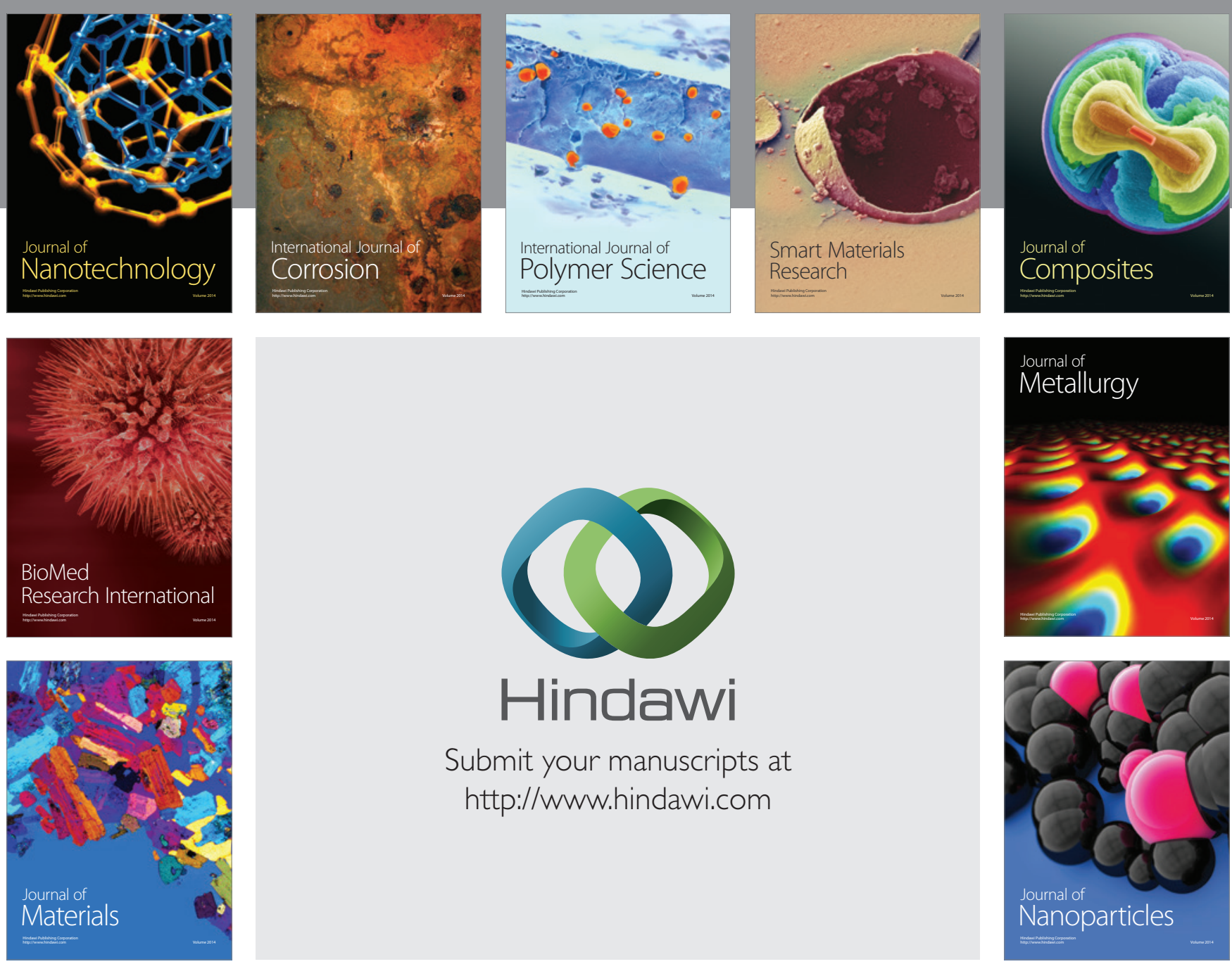

Submit your manuscripts at http://www.hindawi.com
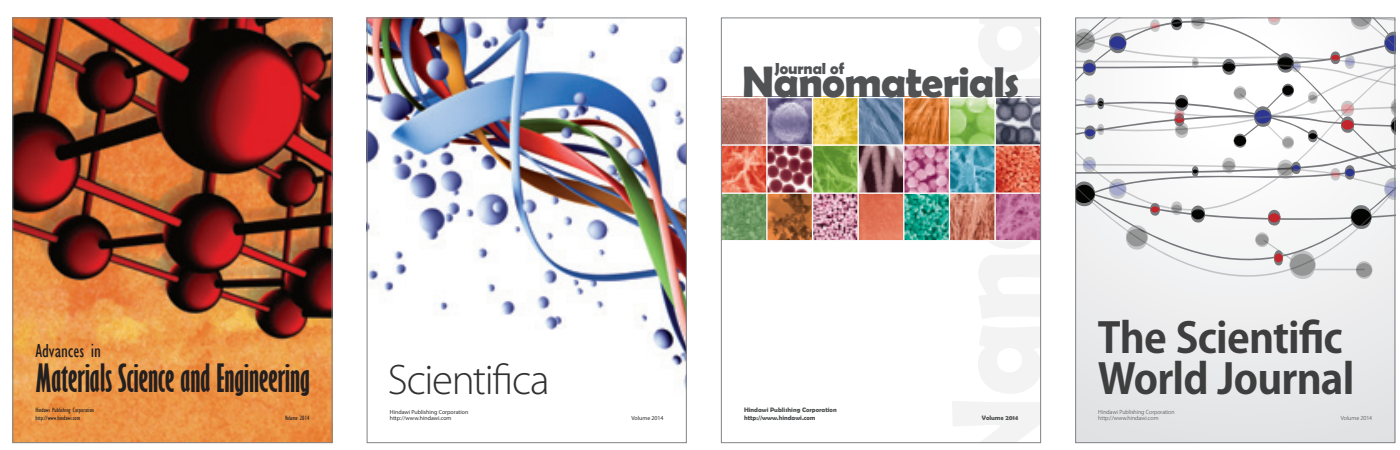

\section{The Scientific World Journal}
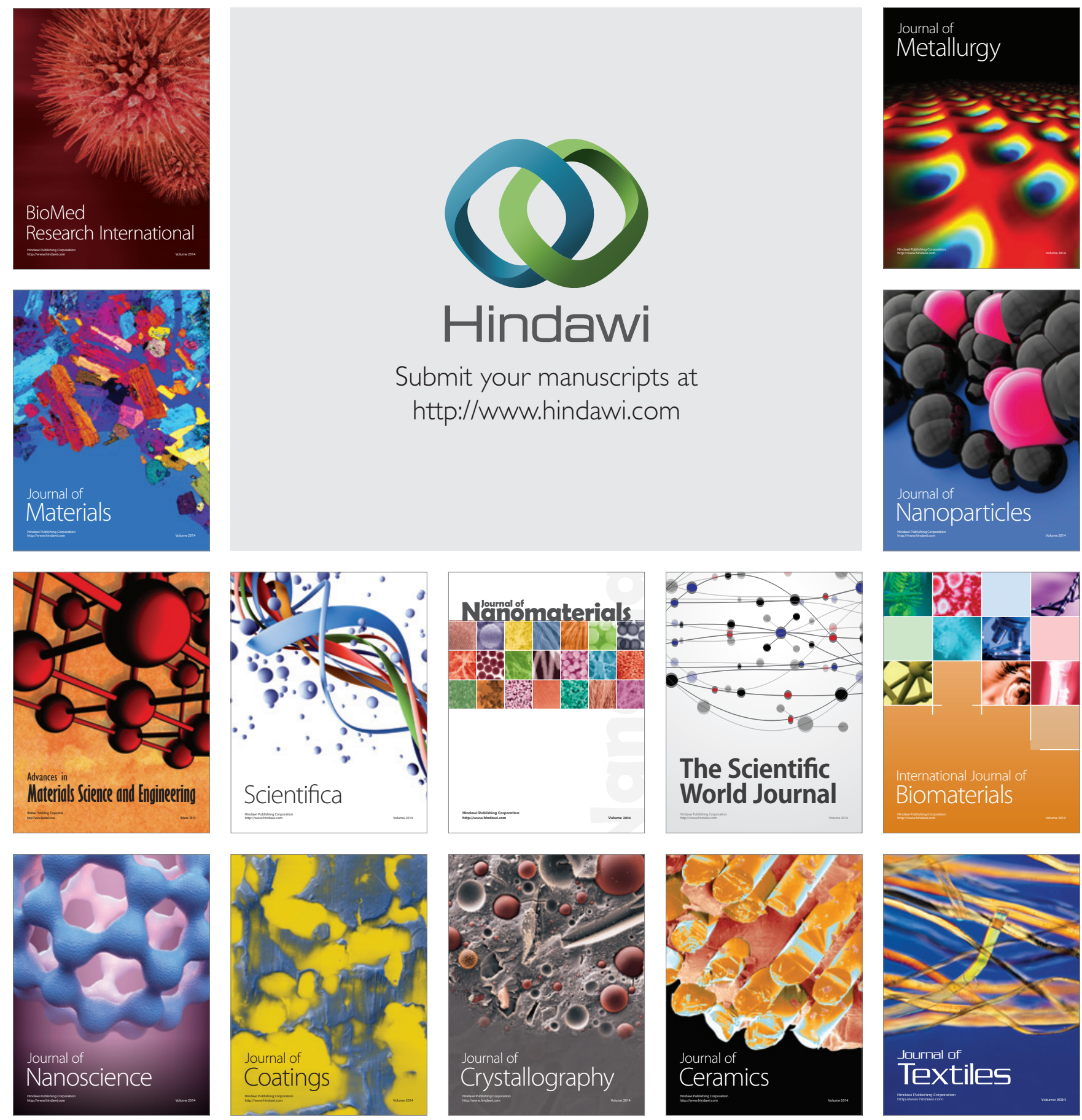